\title{
Water Tunnel Experimental Research on Vortex of Delta Wing
}

\section{Song $\mathrm{LI}^{\mathrm{a}}$, Wei HE ${ }^{\mathrm{b}}$, Kai LUO ${ }^{\mathrm{c}}$, Limin SONG ${ }^{\mathrm{d}}$, Yuankai $\mathrm{LI}^{\mathrm{e}}$, Jicheng ZHANG ${ }^{\dagger}$}

Department of Aviation Theory, Air Force Aviation University, Changchun, 130025, China

alisong4885@126.com, bhewei@163.com, 'luokai@126.com, 'limin@163.com, èuankai@163.com , fjicheng@126.com

Keywords: delta wing; high angle of attack; airflow separate; detached vortices

\begin{abstract}
Flight or maneuvering at high angle of attack is one of the most important parts for the modern fighters. When the angle of attack is within the usual range, the aerodynamic characteristics have a linear relationship with the dynamic of aircraft. With the increase of angle of attack, the airflow separation developed and nonlinear traits of the aerodynamic force and moment occur. Vortex can be generated in front of the nose of the aircraft or leading edge of the wing. Furthermore, the change and break down of the vortices are the main cause for the aerodynamic characteristics at high angle of attack. Study on the structure of the flow field and traits of the vortices at high angle of attack are of great significance to understand the fighter's performance. Through water tunnel experiment, staining flow visualization techniques are used to observe the generation and development of vortex on delta wing. At the same time, for the purpose of studying the effect of sideslip angle, experiments are carried on by adjusting the sideslip angle. It confirms the sideslip makes the flow pattern asymmetrical and the vortex break up at smaller angle of attack.
\end{abstract}

\section{Introduction}

Flight at large angle of attack usually refers to the flight area that flow separation occurs and the aerodynamic present nonlinear characteristics. Early fighter had wings with large aspect ratio (such as rectangular wing), the stalling angle of attack of the wing is very small, within the scope of the use of angle of attack, and lift has a linear relation and the angle of attack. When the Angle of attack is beyond limit, the flow attached on the separation occurs, lift decreases sharply and an adverse rolling moment occurs to make the plane out of control. Therefore, the guideline of the traditional aerodynamic design is attached flow. The purpose is to avoid separated flow separation and vortex, however, a large number of studies have shown that the lee of the wing leading edge separation form vortex can effectively improve the maximum lift, and increase the angle of attack, and thus can enhance the aircraft's mobility. Therefore, the design of the third generation fighter breaks through any limits from original design in the aerodynamic layout. Small aspect ratio and sharp leading edge of the wing make the wings occur flow separation in front under the certain Angle of attack, namely form stable leading edge vortex separation. Lift grows in a nonlinear rate, greatly improving the stalling angle of attack of the aircraft, and increasing the lift-to-drag ratio. Therefore, to carry out research corresponding to the flow field structure and dynamic characteristics at large angle of attack is particularly important.

\section{Basic Flow Pattern and Pressure Distribution of the Delta Wing}

When air flows through the delta wing with the fluctuation of surface pressure, air flow turns up around the side edge of the delta wing and forms detached vortex, and then attaches to the surface again. The air flow transverse outward and then forms the trailing vortex near the trailing edge of the wing, go along the downwash flow direction.

The causes of leading edge vortex and wingtip vortex are similar, but the difference is that the leading edge vortex starts from the front and above the wing. It is very close to the upper surface and interferences the flow pattern obviously and also has a great influence on the lift characteristic; While the wingtip vortex is on lateral margin of the wings, or trailing behind at a distance. Big sweepback delta wing under the small angle of attack and obtuse triangle wing leading edge will 
appear on the leading edge vortex under large angle of attack. When air flows through a delta wing, the air in the boundary layer on the surface of the wing flow to wing leading edge, but due to the large sweepback it cannot pass by the wings; the air on the upper surface of the wing also flow to the wing leading edge. Therefore, two strands of airflow meet with different direction, in this way; the air can't turn up around the wing leading edge like wingtip vortex, but separate on the surface of converging, form free three-dimensional separation vortex without total pressure loss in the leading edge of the wing. Like big sweepback wing, the lateral flow exists on the surface of the delta wings, and the vortex layer is very thin. So, the spiral leading edge vortex with certain strength remains stable and rolls up with the mainstream backward.

Therefore, the conditions of forming stable leading edge vortex can be summarized as the follows: one is free vortex three-dimensional separation at the leading edge can be formed, which can make air produce rotary motion; Second is the mainstream should have enough velocity components along the direction of wing span and thus the vortex is of axial velocity. To satisfy these two conditions, the leading edge of the delta wing is sharp or small round blunt, and the sweepback angle and angle of attack is big enough.

\section{The Influence of Angle of Attack on Detached Vortex}

The experiment was carried on in a water tunnel named HD-2 of low speed aerodynamics laboratory. Experimental model was a delta wing. The experiment was carried on firstly under the condition of zero sideslip, by changing the angle of attack $\left(0^{\circ} \sim 36^{\circ}\right)$ and observes the detached vortex generation and development process. The experimental results are as follows (Fig.1 to Fig.10):

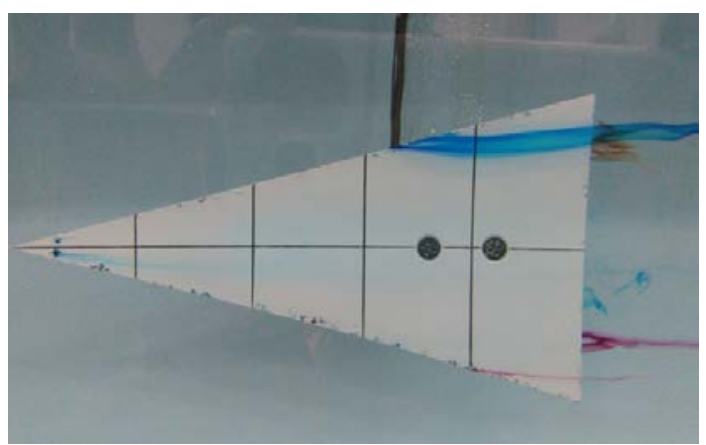

Fig. $1 \mathrm{AOA}=0^{\circ}$

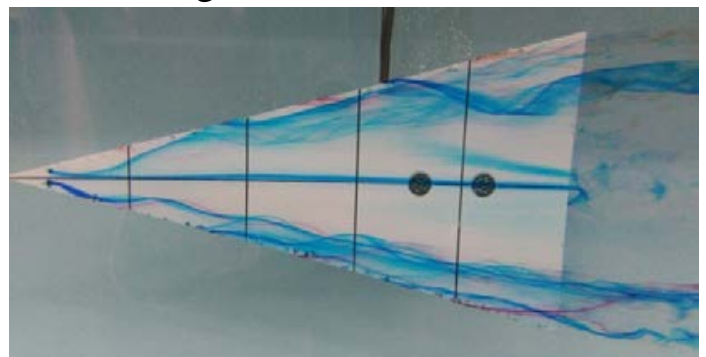

Fig. $3 \quad \mathrm{AOA}=8^{\circ}$

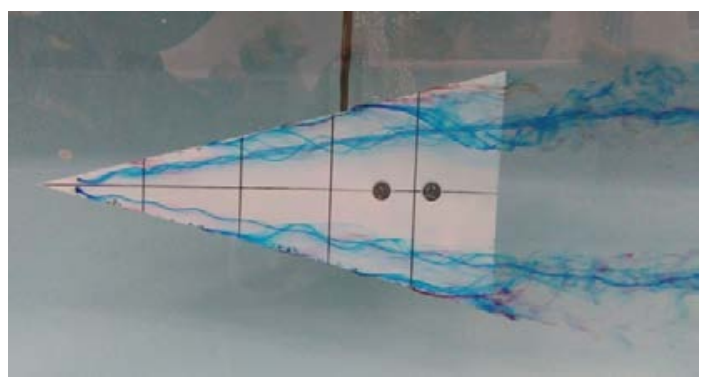

Fig. $5 \quad \mathrm{AOA}=16^{\circ}$

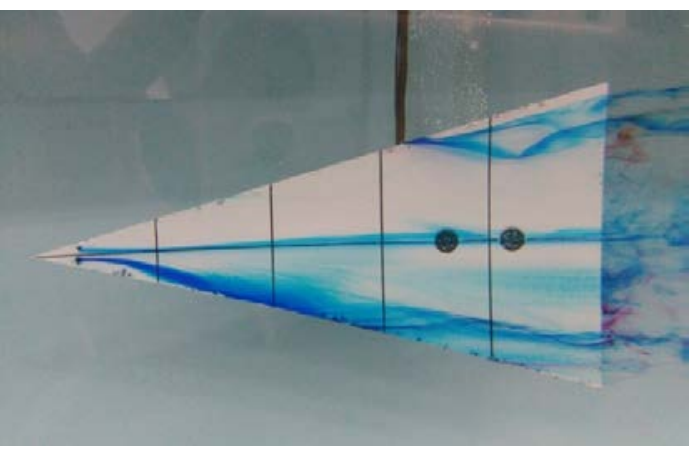

Fig. $2 \mathrm{AOA}=4^{\circ}$

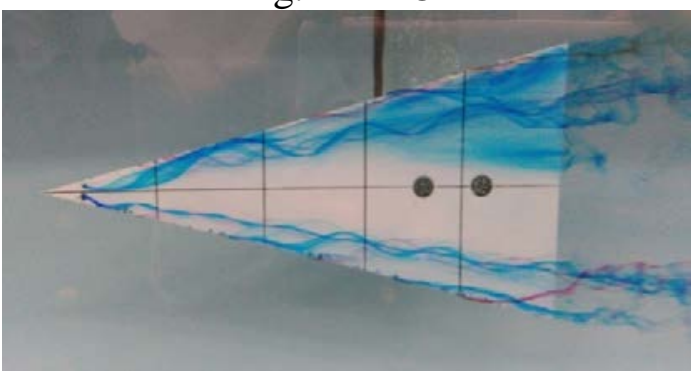

Fig. $4 \mathrm{AOA}=12^{\circ}$

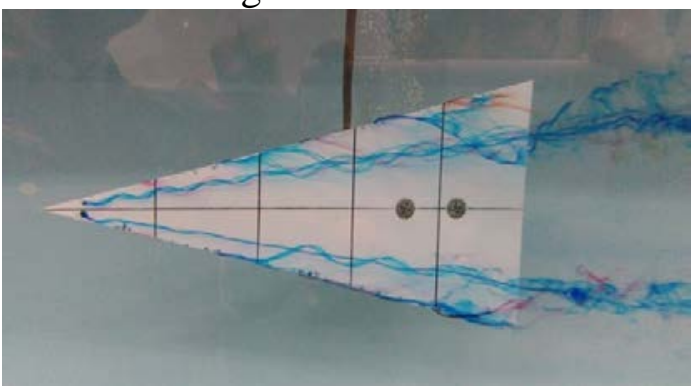

Fig. $6 \quad \mathrm{AOA}=20^{\circ}$ 


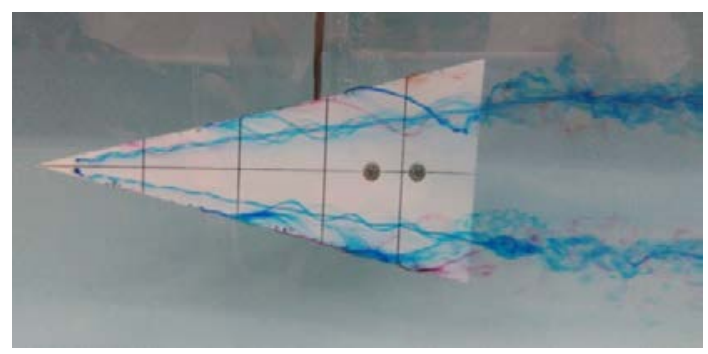

Fig. $7 \quad \mathrm{AOA}=24^{\circ}$

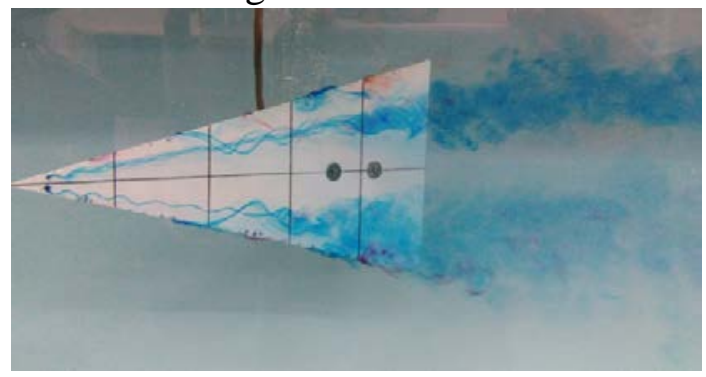

Fig. $9 \quad \mathrm{AOA}=32^{\circ}$

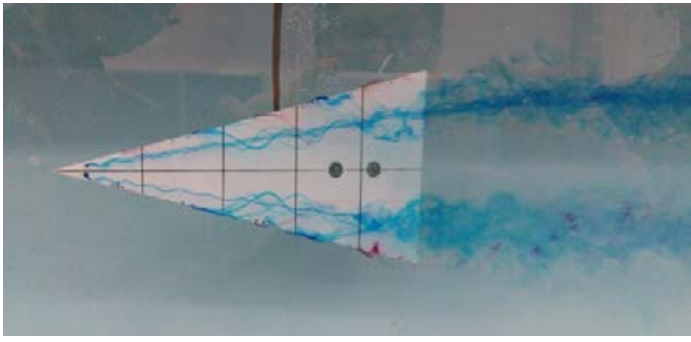

Fig. $8 \quad \mathrm{AOA}=28^{\circ}$

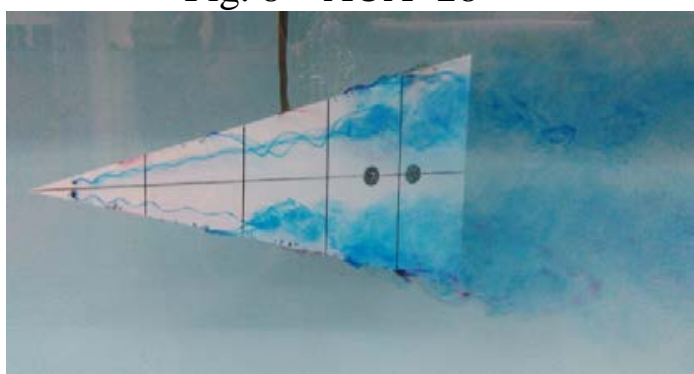

Fig. $10 \mathrm{AOA}=36^{\circ}$

It can be seen from the experimental results that there is no vortex occur at $0^{\circ} \mathrm{AOA}$. When it comes to a $2^{\circ}$ Angle of attack, flow bypass the delta wing edge of the side, turns up colored form detaches vortex flow separation, and then attaches to the surface. It transverse outward, then trailing vortex is formed near the trailing edge of the wing, and go along the downwash flow direction. With the increase of Angle of attack, detached vortex is slowly becoming apparent, and the upper part to take off the body of the development of the vortex is faster than the lower part. When the Angle of attack is $12^{\circ}$, back detached vortex begins to broken, and with the increase of Angle of attack, detached vortex broken slowly forward, at $36^{\circ} \mathrm{AOA}$, detached vortex is almost collapse.

\section{Effect of Sideslip Angle on Detached Vortex}

At $10^{\circ}$ Angle of attack, changing the size of the sideslip Angle (in the range of $2^{\circ}$ to $10^{\circ}$ ), to observe the development and changes of detached vortex. The experimental results are as follows (Fig.11 to Fig.14):

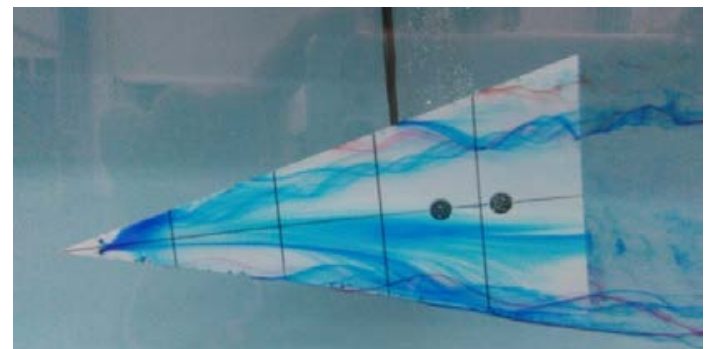

Fig. 11 slip angle $=2^{\circ}$

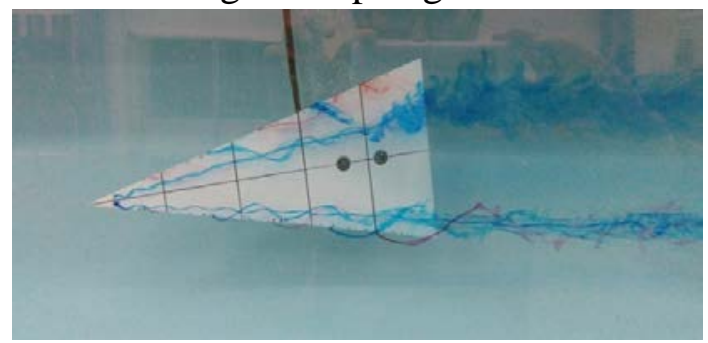

Fig. 13 slip angle $=8^{\circ}$

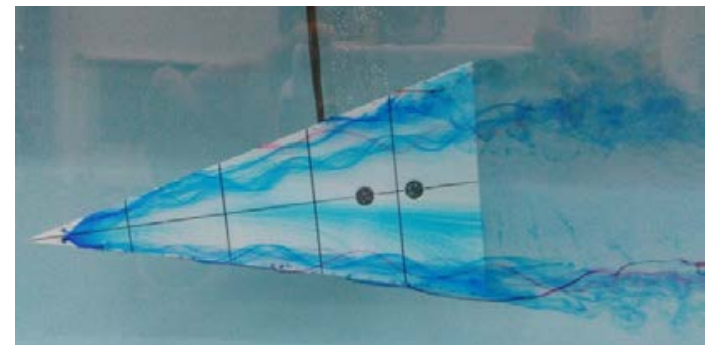

Fig. 12 slip angle $=4^{\circ}$

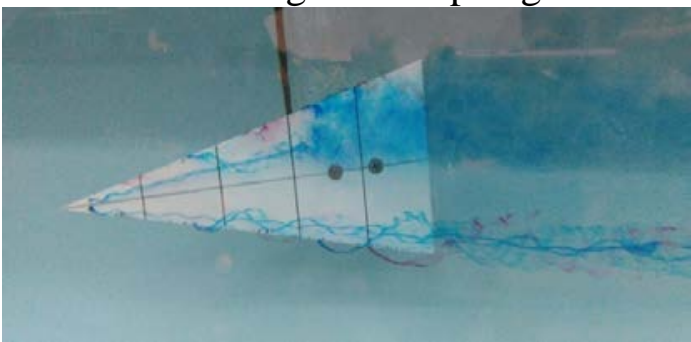

Fig. 14 slip angle $=10^{\circ}$

By experiment, when the sideslip Angle changes, within $2^{\circ} \sim 4^{\circ}$ detached vortex fracture does not occur, but the separation of sideslip direction line shifts inside, lateral spreads outward of a separate line in the opposite direction, detached vortex is no longer symmetry. At the same time, the detached vortex intensity of the front wing is stronger than the other side. With the increase of sideslip Angle, sideslip hind wings detached vortex predated sideslip front wing began to burst. 
When the sideslip Angle reaches $12^{\circ}$, detached vortex of front sideslip wing nearly completely breakdown.

\section{Summary}

This paper analyzes the detached vortex of delta wing theoretically and experimentally. The effects of angle of attack and sideslip on vortex are observed and the main conclusions are as follows:

(1) Detached vortex won't occur when the angle of attack is less than $2^{\circ}$ without sideslip angle. When the AOA is greater than $2^{\circ}$, detached vortex began to occur slowly.

(2) With the increase of Angle of attack, separation point is moving towards and the development of vortex increases. When it comes to a $7^{\circ}$ Angle of attack, the wing surface is in detached vortex.

(3) When the Angle of attack is $12^{\circ}$, detached vortex energy reduces and the vortex starts rupture, and with the development of Angle of attack increases slowly to $30^{\circ}$, detached vortex collapse.

(4) Keep $10^{\circ}$ Angle of attack, with the increase of sideslip Angle, the separation point of the front sideslip wing, the second separation line is moving towards the medial side. And lead to the development of asymmetric vortex. With increasing the sideslip Angle within $2^{\circ}-4^{\circ}$, detached vortex is not split up. When the sideslip Angle is greater than $4^{\circ}$, sideslip front wing detached vortex began to burst, and at $12^{\circ}$ sideslip angle, detached vortex of the front wing collapse.

\section{References}

[1] Qian Yiji, aerodynamics, Beijing university of aeronautics and astronautics press, September 2004.

[2] Fang Zhen ping, flight dynamics, Beijing university of aeronautics and astronautics press, June 2005.

[3] Yang Yonghua , Xu Bangnian, combat aircraft stall spiral pneumatic rotary inertia, the blue sky press, September 1996.

[4] TuLianghui etc., large Angle of attack dynamics analysis and modeling of aviation science and technology between China and Russia colloquium album, in May 2012.

[5] Yang Lizhi, large Angle of attack aerodynamic numerical simulation and modeling studies, xi 'an: northwestern polytechnical university.

[6] Wu Ziniu, aerodynamics, Beijing, tsinghua university press, 2007.

[7] Wang Xinyue, gas dynamic foundation, xi 'an, northwestern polytechnical university press, 2006.

[8] Liu Zhiliang, such as aerodynamics, Beijing, Beijing university of aeronautics and astronautics press, 2009. 